(C) 2016 IEEE. Personal use of this material is permitted. Permission from IEEE must be obtained for all other uses, in any current or future media, including reprinting/republishing this material for advertising or promotional purposes, creating new collective works, for resale or redistribution to servers or lists, or reuse of any copyrighted component of this work in other works. 


\section{Strict Frequency Reuse algorithm in Random Cellular Networks}

\author{
Sinh Cong Lam, Kumbesan Sandrasegaran \\ University of Technology, Sdyney \\ Center of Real Time Information Network \\ Faculty of Engineering and Information Technology \\ Email: sinhcong.lam@student.uts.edu.au; \\ kumbesan.Sandrasegaran@uts.edu.au
}

\author{
Tuan Nguyen Quoc \\ University of Engineering and Technology \\ Vietnam National University, Hanoi \\ Faculty of Electronics and Telecommunication \\ Email:tuannq@vnu.edu.vn
}

\begin{abstract}
A Frequency Reuse algorithm which divides the users into two groups called Cell-Center Users (CCUs) and CellEdge Users (CEUs) is one of the most effective techniques that can mitigate the InterCell Interference as well as improve the spectrum efficiency in cellular networks. In literature, most of work on Strict Frequency Reuse algorithms in a Spatial Point Poisson network assumed that the reuse factor was 1 and there was either single user or single Resource Block (RB). Hence, the performance either $\mathrm{CEU}$ or $\mathrm{CCU}$ was discussed. In this paper, the performance of both types of users as well as total throughput of CEUs and CCUs are investigated. The analytical results indicate that most of users in Tier-1 are served as CCUs while the numbers of UEUs and CCUs in Tier-2 are similar.
\end{abstract}

Index Terms: random cellular network, coverage probability, throughput, strict frequency reuse, Rayleigh-Lognormal.

\section{INTRODUCTION}

In Orthogonal Frequency-Division Multiple Access (OFDMA) multi-cell networks, the main factor that has direct impact on the system performance is intercell interference which is caused by the use of the same frequency band in adjacent cells. InterCell Interference Coordination (ICIC)[1] such as frequency reuse (FR) has been introduced as a technique that can significantly mitigate the intercell interference then improve network performance, especially for users suffering low Signal-to-Interference-plus-Noise ratio (SINR).

Strict Frequency Reuse algorithm is the basic ICIC technique that divides the allocated Resource Blocks (RBs) into $\Delta+1$ groups including one Cell-Center RB group or common $\mathrm{RB}$ group and $\Delta$ Cell-Edge $\mathrm{RB}$ group or private $\mathrm{RB}$ groups. $\Delta$ is called Frequency Reuse Factor. Each cell is allocated a Cell-Center RB group and a Cell-Center RB group. The CellCenter RBs are assigned to CCUs, whose SINRs are greater than a pre-determined SINR threshold. The Cell-Edge RBs are assigned to the rest of CEUs (Cell-Edge Users) whose origin SINRs are smaller than the SINR threshold.

Spatial Point Poisson Process (PPP) has been widely used as an accurate and tractable mathematical model to analyse the performance of cellular network [2]. In [2], [3], the PPP network model was deployed to analyse the performance of the cellular network with single user using Strict Frequency
Reuse with reuse factor of 1 in Rayleigh-Lognormal fading channel.

In [4], [5], the performance of the Strict Frequency Reuse algorithm was evaluated and optimised for the case of multi users. In this paper, the number of users as well as Round Robin scheduling was investigated. However, this paper assumed that the CCU and CEU are served by the same transmit power, thus the difference between a CCU and CEU has not been presented. In the PPP network model using a Frequency Reuse algorithm, authors in [6] indicated that there is always an opposite trend between the performance of CCU and CEU. Hence, analyzing the performance of Cell-Edge and CCU together is a necessary step toward the Frequency Reuse optimization.

In this paper, the performance of the CEU and CCU are considered in the context of a multi-tier network with multiple users and multi-RBs. In the initial state, it is assumed that there is a single user in each area of each cell. The number of new users come to network is assumed to be a Poisson random variable. Each new user is connected to a nearest BS where it is determined as a Cell-Center or CEU based on the received SINR and a SINR threshold.

\section{SYSTEM MODEL}

\section{A. Network topology}

A PPP network model which composes $\mathrm{K}$ tiers is studied in this paper. Each tier is characterised by the density of BSs $\lambda_{k}$ and the standard transmit power $P_{k}$. The downlink signals including desired and interference signals in each tier experience Rayleigh-Lognormal fading with a mean of $\mu_{z}$ and variance $\sigma_{z}$ as well as path loss with exponent $\alpha$.

At initial state, it is assumed that there are $M_{k}$ user in each cell of a tier. The number of new users that request a connection to the network is assumed to be a Poisson random variable with mean $\lambda_{u}$.

Under this network model, the open access protocol where a user is allowed to associate with any tier is studied. The 
probability that a typical user connected to tier- $k$ is given by modifying Lemma 1 in [5]:

$$
A_{k}=\frac{\lambda_{k}}{\sum_{j=1}^{K} \lambda_{j}}
$$

The Probability Density Function (PDF) of the distance $r_{k}$ from a typical user to its nearest BS in tier $k$ is given by [5]

$$
f_{R_{k}}\left(r_{k}\right)=2 \pi\left(\sum_{j=1}^{K} \lambda_{j}\right) r_{k} \exp \left(-\pi r^{2} \sum_{j=1}^{K} \lambda_{j}\right)
$$

In tier- $k$, the average number of new users in a typical cell, $M_{k}$, is defined as the quotient of total number of users and the number of cells. Hence, if the network area is $S, M_{k}$ can be obtained by:

$$
M_{k}=A_{k} \frac{\lambda^{(u)} S}{\lambda_{k} S}=\frac{\lambda^{(u)}}{\sum_{j=1}^{K} \lambda_{j}}
$$

\section{B. Frequency Reuse Algorithm}

In this paper, all cells in tier- $k$ are assumed to use Strict Frequency Reuse with the same reuse factor $\Delta_{k},(0<k \leq K)$ as shown in Figure 1. For example, the resource allocation technique use the SINR threshold $T_{k}$ to divide $M_{k}$ users in each cell into $M_{k}^{(c)}$ CCUs and $M_{k}^{(e)}$ CEUs, $N_{k}$ Resource Blocks (RBs) into $N_{k}^{(c)}$ Cell-Center RBs and $N_{k}^{(e)}$ CellEdge RBs. Furthermore, the Cell-Center RBs are used as the common resources while the Cell-Edge RBs is dived into $\Delta_{k}$ private RB groups. Since, the CCUs do not share their resources with the CEUs, group of CEUs in each cell in tier- $k$ is allocated $N_{k}^{(e)} / \Delta_{k}$ RBs.

In a cellular network system with Round Robin Scheduling, the scheduler randomly allocates a RB from the available RBs to a user. An indicator function $\tau\left(R B_{k}^{(z)}=b\right)$ that can take values 1 if the typical user is served on $\mathrm{RB} b$ in area $z$ of the typical cell in tier- $k$ is defined. $z=c$ or $z=e,(0<k \leq K)$ correspond to a Cell-Center or Cell-Edge area.

Denote $\epsilon_{k}^{(z)}=E\left[\tau\left(R B_{k}^{(z)}=b\right)\right], \epsilon_{k}^{(z)}$ is called the optimization factor or resource allocation ratio and given by:

$$
\epsilon_{k}^{(z)}=\left\{\begin{array}{ll}
1 & \text { if } M_{k}^{(z)}>N_{k}^{(z)} \\
\frac{M_{k}^{(z)}}{N_{k}^{(z)}} & \text { if } M_{k}^{(z)}<N_{k}^{(z)}
\end{array} \quad(\forall 0<k \leq K)\right.
$$

It is assumed that the typical user is served on RB $b$ in tier- $k$. We denote $\theta_{j}^{(c)}$ and $\theta_{j}^{(e)}$ as the set of interfering BSs transmitting on the Cell-Center and Cell-Edge RB in a typical cell in tier $j$. Hence, the densities of BSs in $\theta_{j}^{(c)}$ and $\theta_{j}^{(e)}$ are $\lambda_{j}^{(c)}=\lambda_{j}$ and $\lambda_{j}^{(e)}=\lambda_{j} / \Delta_{j}$ [7]. Hence, the ICI of a typical CCU and CEU are respectively given by:

$$
\begin{gathered}
I_{S t r}^{(c)}=\sum_{z_{c} \in \theta_{j}^{(c)}} \tau\left(R B_{k}^{(c)}=b\right) \tau\left(R B_{j}^{(c)}=b\right) P_{j} g_{j z_{c}} r_{j z_{c}}^{-\alpha} \\
I_{S t r}^{(e)}=\sum_{z_{e} \in \theta_{j}^{(e)}} \tau\left(R B_{k}^{(e)}=b\right) \tau\left(R B_{j}^{(e)}=b\right) \phi_{j} P_{j} g_{j z_{e}} r_{j z_{e}}^{-\alpha}
\end{gathered}
$$

\section{Power}

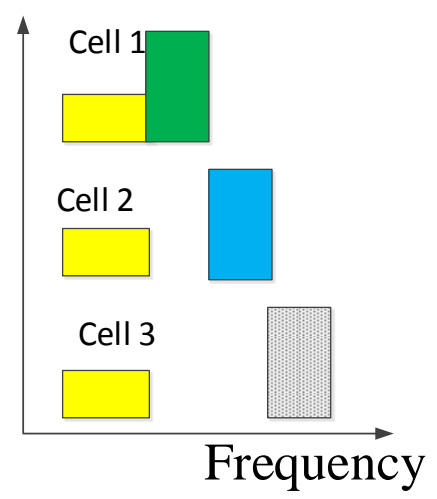

Fig. 1. Strict Frequency Reuse algorithm

in which $g_{j z}$ and $r_{j z}$ are the channel power gain and distance from the user to the interfering $\mathrm{BS} z$ in the tier $j$, $\left(z=\left\{z_{c}, z_{e}\right\}\right)$. In Rayleigh-Lognormal fading channel, the Cumulative Density Function of $g$ is given by[3]

$$
F_{R-L n}(g)=\sum_{n=1}^{N_{H}} \frac{\omega_{n}}{\sqrt{\pi}}\left[1-\exp \left(-\frac{g}{\gamma\left(a_{n}\right)}\right)\right]
$$

in which $\gamma\left(a_{n}\right)=10^{\left(\sqrt{2} \sigma_{z} a_{n}+\mu_{z}\right) / 10} ; w_{n}$ and $a_{n}$ are, respectively, the weights and the abscissas of the Gauss-Hermite polynomial; $\sigma_{z}$ and $\mu_{z}$ are variance and mean of RayleighLognormal Random variable.

\section{Instantaneous SINR}

In the Strict Frequency Reuse network system, the typical user can be served on Cell-Edge RBs with higher transmit power or on Cell-Center RBs with lower transmit power. Hence, the received SINR of a typical user from its serving $\mathrm{BS}$ in tier $k$ can be expressed by following equation:

$S I N R_{k}^{(t)}\left(r_{k}\right)= \begin{cases}\operatorname{SINR}_{k}\left(\phi_{k}, r_{k}\right)=\frac{\phi_{k} P_{k} g_{k} r_{k}^{-\alpha}}{I_{S t r}^{(e)}+\sigma^{2}} & \text { for CEU } \\ \operatorname{SINR}_{k}\left(1, r_{k}\right)=\frac{P_{k} g_{k} r_{k}^{-\alpha}}{I_{\text {Str }}^{(c)}+\sigma^{2}} & \text { for CCU }\end{cases}$

in which $g_{k}$ and $r_{k}$ is the channel power gain and distance from the user to the serving BS in tier $k ; \phi_{k}$ is the transmit power ratio that was defined in section II-B; $\sigma^{2}$ is Gaussian noise; $I_{S t r}^{(c)}$ and $I_{S t r}^{(e)}$ are defined in $5 \mathrm{a}$ and $5 \mathrm{~b}$.

In cellular networks, the Gaussian noise can be neglected compared to the transmit power of BSs, thus in this paper, it was assumed that $\sigma^{2}=0$.

\section{COVERAGE PROBABILITy OF A TYPICAL USER}

In tier- $k$, the CEU is within the coverage region of tier- $k$ if its instantaneous received SINR from its serving BS is greater than the coverage threshold $\hat{T}_{k}$.

$$
\mathbb{P}_{k}^{(e)}\left(\hat{T}_{k}, \phi_{k} \mid r_{k}\right)=\mathbb{P}\left(S I N R_{k}\left(\phi_{k}, r_{k}\right) \geq \hat{T}_{k}\right)
$$


In a mobile network, a typical CEU is within the network coverage if the received $S I N R_{k}\left(\phi_{k}, r_{k}\right)$ from at least one BS in tier $k$ is larger than a coverage threshold $\hat{T}_{k}$.

$$
\begin{aligned}
P_{c}^{(e)} & =\mathbb{P}\left(\bigcup_{k=1}^{K} \operatorname{SINR} R_{k}\left(\phi_{k}, r_{k}\right) \geq \hat{T}_{k}\right) \\
& =\sum_{k=1}^{K} A_{k} \mathbb{P}\left(\operatorname{SINR} R_{k}\left(\phi_{k}, r_{k}\right) \geq \hat{T}_{k}\right) \\
& =2 \pi \sum_{k=1}^{K} \lambda_{k} \int_{0}^{\infty} r_{k} \exp \left(-\pi r_{k}^{2} \sum_{j=1}^{K} \lambda_{j}\right) \mathbb{P}_{k}^{(e)}\left(\hat{T}_{k}, \phi_{k} \mid r_{k}\right) d r_{k}
\end{aligned}
$$

where $\mathbb{P}_{k}^{(e)}\left(\hat{T}_{k}, \phi_{k} \mid r_{k}\right)$ is the coverage probability of the CEU at a distance $r_{k}$ from its serving BS in tier- $k$.

Theorem 3.1: The coverage probability of a CEU

- at a distance $r_{k}$ from its serving BS in the tier $k$

$$
\mathbb{P}_{k}^{(e)}\left(\hat{T}_{k}, \phi_{k} \mid r_{k}\right)=\sum_{n=1}^{N_{H}} \frac{\omega_{n}}{\sqrt{\pi}} e^{-\pi r_{k}^{2} \sum_{j=1}^{K} \frac{\epsilon_{j}^{(e)} \epsilon_{k}^{(e)} \lambda_{j}}{\Delta_{j}} f_{I}^{(e)}\left(\hat{T}_{k}, i, j\right)}
$$

- over tier- $k$

$$
\begin{aligned}
& P_{k}^{(e)}\left(\hat{T}_{k}\right)=\sum_{j=1}^{K} \frac{\lambda_{j}}{\Delta_{j}} \sum_{n=1}^{N_{H}} \frac{\omega_{n}}{\sqrt{\pi}} \frac{1}{\sum_{j=1}^{K} \frac{\lambda_{j}}{\Delta_{j}}\left(1+\epsilon_{j}^{(e)} \epsilon_{k}^{(e)} f_{I}^{(e)}\left(\hat{T}_{k}, i, j\right)\right.} \cdot \\
& \text { over network } \\
& P_{c}^{(e)}\left(\hat{T}_{k}\right)=\frac{\lambda_{k}}{\Delta_{k}} \sum_{n=1}^{N_{H}} \frac{\omega_{n}}{\sqrt{\pi}} \frac{1}{\sum_{j=1}^{K} \frac{\lambda_{j}}{\Delta_{j}}\left(1+\epsilon_{j}^{(e)} \epsilon_{k}^{(e)} f_{I}^{(e)}\left(\hat{T}_{k}, i, j\right)\right)}
\end{aligned}
$$

where $f_{I}^{(e)}\left(\hat{T}_{k}, i, j\right)=$

$$
\sum_{n_{1}=1}^{N_{H}} \frac{\omega_{n_{1}}}{\sqrt{\pi}}\left[\frac{2 \pi C\left(\hat{T}_{k}, \phi_{k}\right)^{\frac{2}{\alpha}}}{\alpha \sin \left(\frac{\pi(\alpha-2)}{\alpha}\right)}-\sum_{m=1}^{N_{L}} \frac{c_{m}}{2} \frac{C\left(\hat{T}_{k}, \phi_{k}\right)}{c_{m}+\left(\frac{x_{m}+1}{2}\right)^{\alpha / 2}}\right] ;
$$

$C\left(\hat{T}_{k}, \phi_{k}\right)=\hat{T}_{k} \frac{\gamma\left(a_{n_{1}}\right)}{\gamma\left(a_{n}\right)} \frac{\phi_{j} P_{j}}{\phi_{k} P_{k}} ; \gamma\left(a_{n}\right)=10^{\left(\sqrt{2} \sigma_{z} a_{n}+\mu_{z}\right) / 10} ;$ $\omega_{n}$ and $a_{n}, c_{m}$ and $x_{m}$ are are weights and nodes of GaussHermite, Gauss-Legendre rule respectively with order $N_{H}$.

Proof: See Appendix

There are two main differences between a CEU and CCU in Strict Frequency Reuse. The first difference relates to the power of the serving signal. The CEU is served on CE RB at a higher power level, i.e. $\phi_{k} P_{k}$, while the CCU is served on CC $\mathrm{RB}$ at a lower power level, i.e. $P_{k}$. The second difference relates to the interference signal. The BSs in tier- $j$ cause interference to the CEU and CCU transmitting at power $\phi_{j} P_{j}$ and $P_{j}$, respectively. Hence, the coverage probability of the typical CCU at a distance $r_{k}$ from its serving BS and average coverage probability when it connects to a BS in tier- $k$ can be obtained by:

$$
\mathbb{P}_{k}^{(c)}\left(\hat{T}_{k}, 1 \mid r_{k}\right)=\sum_{n=1}^{N_{H}} \frac{\omega_{n}}{\sqrt{\pi}} e^{-\pi r_{k}^{2} \sum_{j=1}^{K} \epsilon_{j}^{(c)} \epsilon_{k}^{(c)} \lambda_{j} f_{I}^{(c)}\left(\hat{T}_{k}, i, j\right)}
$$

and

$$
P_{c}^{(c)}\left(\hat{T}_{k}\right)=\sum_{n=1}^{N_{H}} \frac{\omega_{n}}{\sqrt{\pi}} \frac{\sum_{j=1}^{K} \lambda_{j}}{\sum_{j=1}^{K} \lambda_{j}\left(1+\epsilon_{j}^{(k)} \epsilon_{k}^{(c)} f_{I}^{(c)}\left(\hat{T}_{k}, i, j\right)\right)}
$$

in which $f_{I}^{(c)}\left(\hat{T}_{k}, i, j\right)=$

$$
\sum_{n_{1}=1}^{N_{H}} \frac{\omega_{n_{1}}}{\sqrt{\pi}}\left[\frac{2 \pi C\left(\hat{T}_{k}, 1\right)^{\frac{2}{\alpha}}}{\alpha \sin \left(\frac{\pi(\alpha-2)}{\alpha}\right)}-\sum_{m=1}^{N_{L}} \frac{c_{m}}{2} \frac{C\left(\hat{T}_{k}, 1\right)}{c_{m}+\left(\frac{x_{m}+1}{2}\right)^{\alpha / 2}}\right] ;
$$

$C\left(\hat{T}_{k}, \phi_{k}\right)=\hat{T}_{k} \frac{\gamma\left(a_{n_{1}}\right)}{\gamma\left(a_{n}\right)} \frac{P_{j}}{P_{k}}$; the related symbols are defined in Theorem 3.1.

Theorem 3.2: The arrival of a new user within the PPP network is represented as a Poisson random variable with mean $\lambda^{(u)}$. The number of new users which are served as CEU and CCUs are given by

- new CCUs

$$
M_{k}^{(c)}=\lambda^{(u)} \sum_{n=1}^{N_{H}} \frac{\omega_{n}}{\sqrt{\pi}} \frac{1}{\sum_{j=1}^{K} \lambda_{j}\left(1+\epsilon_{j}^{(o c)} \epsilon_{k}^{(o c)} f_{I}^{(o e)}\left(T_{k}, i, j\right)\right)}
$$

where $f_{I}^{(c)}\left(\hat{T}_{k}, i, j\right)=$ $\sum_{n_{1}=1}^{N_{H}} \frac{\omega_{n_{1}}}{\sqrt{\pi}}\left[\frac{2 \pi C\left(T_{k}, 1\right)^{\frac{2}{\alpha}}}{\alpha \sin \left(\frac{\pi(\alpha-2)}{\alpha}\right)}-\sum_{m=1}^{N_{L}} \frac{c_{m}}{2} \frac{C\left(T_{k}, 1\right)}{c_{m}+\left(\frac{x_{m}+1}{2}\right)^{\alpha / 2}}\right]$; new CEUs

$$
\begin{aligned}
& M_{k}^{(e)}=M_{k}-M_{k}^{(c)} \\
& =\frac{\lambda^{(u)}}{\sum_{j=1}^{K} \lambda_{j}}-\sum_{n=1}^{N_{H}} \frac{\omega_{n}}{\sqrt{\pi}} \frac{\lambda^{(u)}}{\sum_{j=1}^{K} \lambda_{j}\left(1+\epsilon_{j}^{(o c)} \epsilon_{k}^{(o c)} f_{I}^{(o e)}\left(\hat{T}_{k}, i, j\right)\right)}
\end{aligned}
$$

Proof: The user is considered a CCU in tier- $k$ if it connect to tier $\mathrm{k}$ and its original SINR at initial state is smaller than the threshold. Hence, the probability where the user is defined as a $\mathrm{CCU}$ is

$$
\begin{aligned}
\mathbb{P}_{a s-k}^{(e)} & =\mathbb{P}\left(S I N R_{k}^{(o)}\left(1, r_{k}\right)>T_{k} \mid u=k\right) \\
& =\frac{\mathbb{P}\left(S I N R_{k}^{(o)}\left(1, r_{k}\right)>T_{k}, u=k\right)}{\mathbb{P}(u=k)}
\end{aligned}
$$

in which $u=k$ is the event that the user connect to tier- $k$. $\mathbb{P}(u=k)$ is defined in Equation 1 .

In Equation 17, the denominator represents the probability that a user is associated with tier- $k$ and is given by $A_{k}$ in Equation 1. The joint probability in the numerator is evaluated using the following steps:

$$
\begin{aligned}
& \mathbb{P}\left(\operatorname{SINR}_{k}^{(o)}\left(1, r_{k}\right)>T_{k}, u=k\right) \\
& =\mathbb{P}\left(S I N R_{k}^{(o)}\left(1, r_{k}\right)>T_{k}, r_{k}>\max _{j \neq k} r_{j}\right) \\
& =\int_{0}^{\infty} \mathbb{P}\left(S I N R_{k}^{(o)}\left(1, r_{k}\right)>T_{k}, r_{k}>\max _{j \neq k} r_{j}\right) g_{R_{k}}\left(r_{k}\right) d r_{k} \\
& =\int_{0}^{\infty} \mathbb{P}\left(\operatorname{SINR}_{k}^{(o)}\left(1, r_{k}\right)>T_{k}\right) \mathbb{P}\left(r_{k}>\max _{j \neq k} r_{j}\right) g_{R_{k}}\left(r_{k}\right) d r_{k}
\end{aligned}
$$


in which $g_{R_{k}}\left(r_{k}\right)=2 \pi \lambda_{k} r_{k} \exp \left(-\pi \lambda_{k} r_{k}^{2}\right)$ is the PDF of the distance from the user to the BS in tier- $k$.

The first element of the integrand in Equation 18 can be evaluated as Appendix A, i.e $\mathbb{P}\left(\frac{P_{k} g_{k} r_{k}^{-\alpha}}{I_{S t r}^{(c)}>T_{k}}\right)=\mathbb{P}\left(T_{k}, 1 \mid r_{k}\right)$. The second element is the Cumulative Density Function (PDF) of the distance from the user to its serving BS and can be evaluated by using the properties of the Null probability of a 2-D Poisson process .

$$
\begin{aligned}
\mathbb{P}\left(r_{k}<\min _{j \neq k}\left(r_{j}\right)\right) & =\prod_{j=k, j \neq k}^{K} \mathbb{P}\left(r_{k}<r_{j}\right) \\
& =\prod_{j=k, j \neq k}^{K} \mathbb{P}\left(r_{j}>r_{k}\right) \\
& =\exp \left(-\pi \sum_{j=k, j \neq k}^{K} \lambda_{j} r_{k}^{2}\right)
\end{aligned}
$$

Subsequently, the number of CCUs in a typical cell in tier- $k$ is given by

$$
\begin{aligned}
M_{k}^{(c)} & =M_{k} \frac{\mathbb{P}\left(S I N R_{k}^{(o)}\left(1, r_{k}\right)<T, u=k\right)}{\mathbb{P}(u=k)} \\
& =2 \pi \lambda^{(u)} \int_{0}^{\infty} r_{k} \mathbb{P}^{(o)}\left(T_{k}, 1 \mid r_{k}\right) \exp \left(-\pi \sum_{j=1}^{K} \lambda_{j} r_{k}^{2}\right) d r_{k} \\
& =\lambda^{(u)} \sum_{n=1}^{N_{H}} \frac{\omega_{n}}{\sqrt{\pi}} \frac{1}{\sum_{j=1}^{K} \lambda_{j}\left(1+\epsilon_{j}^{(o c)} \epsilon_{k}^{(o c)} f_{I}^{(o e)}\left(T_{k}, i, j\right)\right)}
\end{aligned}
$$

\section{CAPACITY}

In this section, the average throughputs of Cell-Center and Cell-Edge area of a typical cell in tier- $k$ which are defined as the total average throughput of all users in the corresponding areas are presented. The throughput of a typical Cell-Edge area in tier- $k$ is given by

$$
C_{C E A}^{(e)}=M_{k}^{(e)} C_{k}^{(e)}
$$

in which $M_{k}^{(e)}$ is the average number of CEUs in a typical cell in tier- $k$ and obtained from $3.2 ; C_{k}^{(e)}$ is the average data rate of the $\mathrm{CEU}$ in tier- $k$.

Hence, in order to compute the average throughput of CellEdge area in tier- $k$, the average throughput of the CEU in this tier should be evaluated first. Using the Shannon theorem, $C_{k}^{(e)}$ is given by

$$
C_{k}^{(e)}=\mathbb{E}\left(\ln \left(S I N R\left(\phi_{k}, r_{k}\right)+1\right)\right)
$$

in which $\operatorname{SINR}\left(\phi_{k}, r_{k}\right)$ is SINR at the CEU and defined in Equation 7.

$$
\text { Since } \begin{aligned}
\mathbb{E}(X) & =\int_{t>0} \mathbb{P}(X>t), \quad \forall X>0, \\
C_{k}^{(e)} & =\int_{0}^{\infty} \mathbb{E}\left(\ln \left(\operatorname{SINR}\left(\phi_{k}, r_{k}\right)+1\right)>t\right) d t \\
& =\int_{0}^{\infty} \mathbb{P}\left(\operatorname{SINR}\left(\phi_{k}, r_{k}\right)>e^{t}-1\right) d t \\
& =\int_{0}^{\infty} P_{c}^{(e)}\left(e^{t}-1\right) d t
\end{aligned}
$$

in which $P_{c}^{(e)}\left(e^{t}-1\right)$ is the average coverage probability of the CEU in the PPP network and is obtained by Equation 12. Subsequently, the average throughput of the Cell-Edge area in tier- $k$ is given by

$$
\begin{aligned}
C_{C E A}^{(e)}= & \left(\frac{\lambda^{(u)}}{\sum_{j=1}^{K} \lambda_{j}}-\sum_{n=1}^{N_{H}} \frac{\omega_{n}}{\sqrt{\pi}} \frac{\lambda^{(u)}}{\sum_{j=1}^{K} \lambda_{j}\left(1+\epsilon_{j}^{(c)} \epsilon_{k}^{(c)} f_{I}^{(e)}\left(T_{k}, i, j\right)\right)}\right) \\
& \sum_{n=1}^{N_{H}} \frac{\omega_{n}}{\sqrt{\pi}} \int_{0}^{\infty} \frac{\sum_{j=1}^{K} \frac{\lambda_{j}}{\Delta_{j}}}{\sum_{j=1}^{K} \frac{\lambda_{j}}{\Delta_{j}}\left(1+\epsilon_{j}^{(e)} \epsilon_{k}^{(e)} f_{I}^{(e)}\left(e^{t}-1, i, j\right)\right)} d t
\end{aligned}
$$

Similarity, the average throughput of the Cell-Center area in tier- $k$ is

$$
\begin{aligned}
C_{C C A}^{(c)}= & \lambda^{(u)} \sum_{n=1}^{N_{H}} \frac{\omega_{n}}{\sqrt{\pi}} \frac{1}{\sum_{j=1}^{K} \lambda_{j}\left(1+\epsilon_{j}^{(c)} \epsilon_{k}^{(c)} f_{I}^{(e)}\left(T_{k}, i, j\right)\right)} \\
& \sum_{n=1}^{N_{H}} \frac{\omega_{n}}{\sqrt{\pi}} \int_{0}^{\infty} \frac{\sum_{j=1}^{K} \lambda_{j}}{\sum_{j=1}^{K} \lambda_{j}\left(1+\epsilon_{j}^{(c)} \epsilon_{k}^{(c)} f_{I}^{(c)}\left(e^{t}-1, i, j\right)\right)} d t
\end{aligned}
$$

\section{Simulation and Discussion}

\section{A. Simulation setup}

In the simulation work, it was assumed that each cell in the network is allocated $20 \mathrm{RBs}$ in which the Cell-Edge and CellCenter area are allowed 20 and 10 RBs respectively. In initial state, it was assumed that there is one user in Cell-Center area and one user in Cell-Edge area of each tier. The number of new user arrivals is a Poisson random variable with a mean of $\lambda^{(u)}$.

The analytical and simulation parameters used in this paper are summarized in Table I.

Figure 2 presents variation of the average coverage probability of the CCU and CEU (y-axis) at initial state of the network where there is a single user in the Cell-Edge and Cell-Center area.

It is observed from Figure 2 that the average coverage probability of the CCU is significantly greater than that of the CEU. For example, when the coverage threshold is 0 $\mathrm{dB}$, the average coverage probability of the $\mathrm{CCU}$ is around 


\begin{tabular}{|l|l|}
\hline Parameter & Value \\
\hline Number of tiers & $K=2$ \\
\hline Density of BSs & $\begin{array}{l}\text { Tier } 1, \lambda=0.25 \\
\text { Tier } 2, \lambda=0.5\end{array}$ \\
\hline Transmit power & $P_{1}=100$ \\
- Tier 1 & $P_{2}=1$ \\
- Tier 2 & $\phi_{1}=20$ \\
\hline Transmit power ratio & $\phi_{2}=10$ \\
- Tier 1 & $\Delta_{1}=\Delta_{2}=3$ \\
- Tier 2 & $T_{1}=T_{2}=10 \mathrm{~dB}$ \\
\hline Frequency reuse factor & $\mu_{z}=-7.3683 \mathrm{~dB}$ \\
\hline SINR threshold & $\sigma_{z}=8 \mathrm{~dB}$ \\
\hline Fading channel & $\alpha=4$ \\
\hline Pathloss exponent & $\alpha$ \\
\hline
\end{tabular}

ANALYTICAL AND SIMULATION PARAMETERS

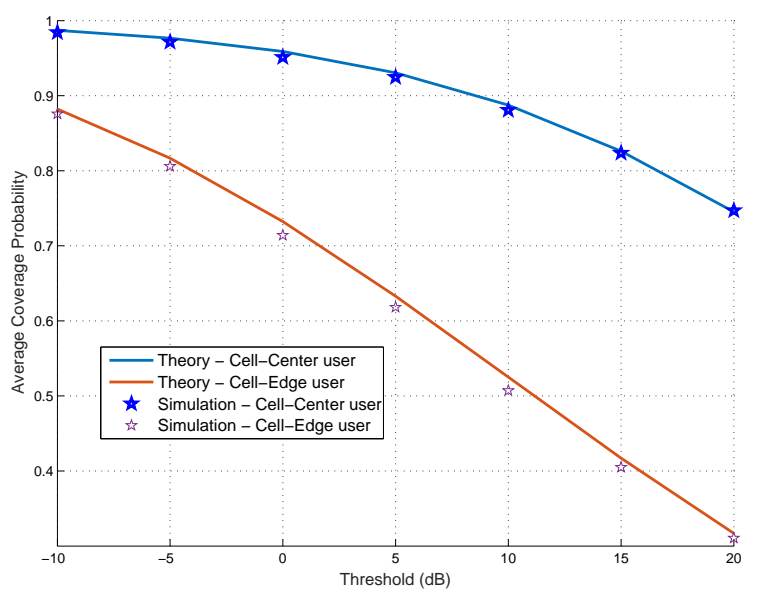

Fig. 2. Coverage probability of a Cell-Center and Cell-Edge user vs. coverage threshold

0.9511 which is approximately $23 \%$ greater than that of CEU. This because when Strict Frequency Reuse with factor $\Delta_{1}=\Delta_{2}=3$ is deployed, the number of RBs in Cell-Edge in this case is only 7 while the Cell-Center area is allocated 10 RBs.

It is observed from Figure 3 and 4 that most of users in Tier- 1 are served as CCUs. Meanwhile the number of CEU and CCUs in Tier-2 are similar. This is because the users in Tier-1 are served by BSs with higher transmit power which can combat the InterCell Interference.

Figure 4 indicates that the throughput of CCU in Tier-1 is significantly higher than those of other areas. For example, when density of new users is 6 , the throughput of CCU in Cell-Center area in Tier-1 is double that in Tier-2. Hence, it is proposed that this area can be used to serve the users that require a higher data rate.

\section{CONCLUSION}

In this paper, the performance of CEU and CCU in a PPP network with multi-user and multi-RB was presented. The

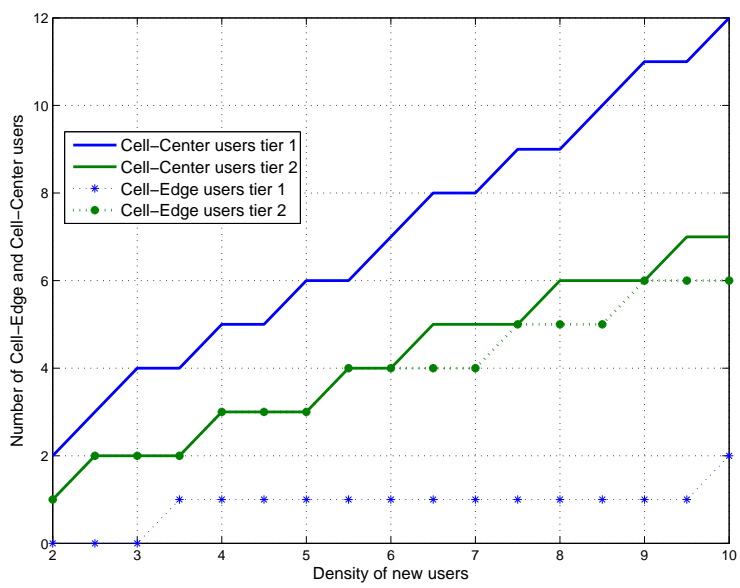

Fig. 3. Number of new Cell-Edge and CCUs in each tier

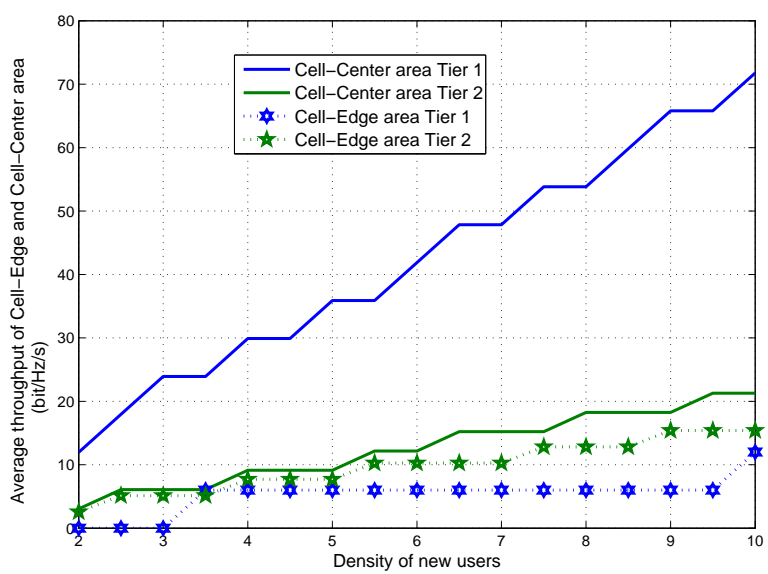

Fig. 4. Average throughput of Cell-Center and Cell-Edge area

numerical analysis indicates that the performance of the CCU is significantly better than that of CEU. Furthermore, it was observed that most of users are served as CCUs in Tier-1 which has higher transmit power while there is not much difference between the number of CEUs and CCUs in Tier2 which has lower transmit power. Hence, for optimization purpose, the allocation algorithm should be designed to tradeoff between the performance of Cell-Edge area and CellCenter area in each tier.

\section{APPENDIX A}

The coverage probability of the user which served on Cell-Edge RB with Signal-to-Interference-Noise Ratio 
$S I N R_{k}\left(\phi_{k}, r_{k}\right)$ is defined as

$$
\begin{aligned}
\mathbb{P}_{c}^{(e)}\left(\hat{T}_{k}, \phi_{k} \mid r_{k}\right) & =\mathbb{P}\left(S I N R_{k}\left(\phi_{k}, r_{k}\right)>\hat{T}_{k}\right) \\
& =\sum_{n=1}^{N_{H}} \frac{\omega_{n}}{\sqrt{\pi}} \mathbb{E}\left[\exp \left(-\frac{\hat{T}_{k} r_{k}^{\alpha}\left(I_{u}+\sigma^{2}\right)}{\phi_{k} P_{k} \gamma\left(a_{n}\right)}\right)\right] \\
& =\sum_{n=1}^{N_{H}} \frac{\omega_{n}}{\sqrt{\pi}} \mathbb{E}\left\{\exp \left(-\frac{T r_{k}^{\alpha} I_{u}}{\phi_{k} P_{k} \gamma\left(a_{n}\right)}\right)\right\}
\end{aligned}
$$

The expectation can be evaluated by following step:

$$
\begin{aligned}
& \mathbb{E}\left\{\exp \left(-\frac{\hat{T}_{k} r_{k}^{\alpha}}{\phi_{k} P_{k} \gamma\left(a_{n}\right)} I_{u}\right)\right\} \\
& =\mathbb{E}\left\{\exp \left(-\frac{\hat{T}_{k} \phi_{j} P_{j}}{\phi_{k} P_{k} \gamma\left(a_{n}\right)} \tau\left(R B_{k}^{(e)}=b\right) \tau\left(R B_{j}^{(e)}=b\right) g_{j z_{c}} \frac{r_{j z_{c}}^{-\alpha}}{r_{k}^{-\alpha}}\right)^{\text {in }} P_{k}^{(e)}\right. \\
& =\prod_{j=1}^{K} \mathbb{E}\left\{\prod_{z_{e} \in \theta_{j}^{(e)}} \epsilon_{j}^{(e)} \epsilon_{k}^{(e)} \mathbb{E}_{g_{j z}}\left[\exp \left(-C\left(\hat{T}_{k}, \phi_{k}\right) g_{j z_{e}}\left(\frac{r_{j z_{e}}}{r_{k}}\right)^{-\alpha}\right)^{k}\right]\right.
\end{aligned}
$$

in which $C\left(\hat{T}_{k}, \phi_{k}\right)=\hat{T}_{k} \frac{\gamma\left(a_{n_{1}}\right)}{\gamma\left(a_{n}\right)} \frac{\phi_{j} P_{j}}{\phi_{k} P_{k}}$.

Since $g_{j z_{c}}$ is Rayleigh-Lognormal fading channel then

$=\prod_{j=1}^{K} \mathbb{E}\left\{\prod_{z_{e} \in \theta_{j}^{(c)}} \epsilon_{j}^{(e)} \epsilon_{k}^{(e)} \sum_{n_{1}=1}^{N_{H}} \frac{\omega_{n_{1}}}{\sqrt{\pi}} \frac{1}{1+C\left(\hat{T}_{k}, \phi_{k}\right)\left(r_{j z_{e}} / r_{k}\right)^{-\alpha}}\right\}$

Each element of this product can be evaluated by using the properties of PPP generating function. Hence, the expectation equals:

$$
=\prod_{j=1}^{K} \exp \left(-2 \pi \lambda_{j}^{(e)} \int_{r_{k}}^{\infty} 1-\frac{1}{1+C\left(\hat{T}_{k}, \phi_{k}\right)\left(r_{j z_{e}} / r_{k}\right)^{-\alpha}}\right) d r_{j z}
$$

Employing the changes in variable $t=\left(\frac{r_{j z_{e}}}{r_{k}}\right)^{2}$, the expectation becomes.

$$
\begin{aligned}
=\prod_{j=1}^{K} \exp \left\{-\pi \lambda_{j}^{(e)} r_{k}^{2}\right. & \left(\int_{0}^{\infty} \frac{C\left(\hat{T}_{k}, \phi_{k}\right)}{1+C\left(\hat{T}_{k}, \phi_{k}\right) t^{-\alpha / 2}} d t\right. \\
& \left.\left.-\int_{0}^{1} \frac{C\left(\hat{T}_{k}, \phi_{k}\right)}{1+C\left(\hat{T}_{k}, \phi_{k}\right) t^{-\alpha / 2}} d t\right)\right\}
\end{aligned}
$$

The first integral can be evaluated by using Gamma function, the second integral can be approximated by using GaussLegendre approximation quadrature as shown in [3], then the expectation equals

$$
\mathbb{E}^{(e)}=\exp \left(-\pi r_{k}^{2} \sum_{j=1}^{K} \frac{\epsilon_{j}^{(e)} \epsilon_{k}^{(e)} \lambda_{j}}{\Delta_{j}} f_{I}^{(e)}\left(\hat{T}_{k}, i, j\right)\right)
$$

in which

$$
\begin{aligned}
f_{I}^{(e)}\left(\hat{T}_{k}, i, j\right)= & \sum_{n_{1}=1}^{N_{H}} \frac{\omega_{n_{1}}}{\sqrt{\pi}}\left(\frac{2 \pi C\left(\hat{T}_{k}, \phi_{k}\right)^{\frac{2}{\alpha}}}{\alpha \sin \left(\frac{\pi(\alpha-2)}{\alpha}\right)}\right. \\
& \left.-\sum_{m=1}^{N_{L}} \frac{c_{m}}{2} \frac{C\left(\hat{T}_{k}, \phi_{k}\right)}{c_{m}+\left(\frac{x_{m}+1}{2}\right)^{\alpha / 2}}\right)
\end{aligned}
$$

where $c_{m}$ and $x_{m}$ are are weights and nodes of GaussLegendre rule with order $N_{L}$.

Hence, the coverage probability $\mathbb{P}_{c}^{(e)}\left(\hat{T}_{k}, \phi_{k} \mid r_{k}\right)$ equals

$$
\mathbb{P}_{c}^{(e)}\left(\hat{T}_{k}, \phi_{k} \mid r_{k}\right)=\sum_{n=1}^{N_{H}} \frac{\omega_{n}}{\sqrt{\pi}} \exp \left(-\pi r_{k}^{2} \sum_{j=1}^{K} \frac{\epsilon_{j}^{(e)} \epsilon_{k}^{(e)} \lambda_{j}}{\Delta_{j}} f_{I}^{(e)}\left(\hat{T}_{k}, i, j\right)\right)
$$

The average coverage probability of the CEU when it connects to tier- $k$ is

$$
P_{k}^{(e)}\left(\hat{T}_{k}\right)=\int_{0}^{\infty} \mathbb{P}_{c}^{(e)}\left(\hat{T}_{k}, \phi_{k} \mid r_{k}\right) f_{R_{k}}\left(r_{k}\right) d r_{k}
$$

in which $f_{R_{k}}\left(r_{k}\right)$ is the PDF of the distance from the user to ts serving BS and defined in Equation 2. Then,

$$
\}_{k}^{k}\right\}\left\{\hat{T}_{k}\right)=\sum_{j=1}^{K} \frac{\lambda_{j}}{\Delta_{j}} \sum_{n=1}^{N_{H}} \frac{\omega_{n}}{\sqrt{\pi}} \frac{1}{\sum_{j=1}^{K} \frac{\lambda_{j}}{\Delta_{j}}\left(1+\epsilon_{j}^{(e)} \epsilon_{k}^{(e)} f_{I}^{(e)}\left(\hat{T}_{k}, i, j\right)\right)}
$$

The average coverage probability in network is given by

$$
P_{k}^{(e)}\left(\hat{T}_{k}\right)=\frac{\lambda_{k}}{\Delta_{k}} \sum_{n=1}^{N_{H}} \frac{\omega_{n}}{\sqrt{\pi}} \frac{1}{\sum_{j=1}^{K} \frac{\lambda_{j}}{\Delta_{j}}\left(1+\epsilon_{j}^{(e)} \epsilon_{k}^{(e)} f_{I}^{(e)}\left(\hat{T}_{k}, i, j\right)\right)}
$$

\section{REFERENCES}

[1] Soft Frequency Reuse Scheme for UTRAN LTE, 3GPP Project Document Std. R1-050 507, Huawei,2005.

[2] J. G. Andrews, F. Baccelli, and R. K. Ganti, "A new tractable model for cellular coverage," in Communication, Control, and Computing (Allerton), 2010 48th Annual Allerton Conference on, Conference Proceedings, pp. 1204-1211.

[3] S. C. Lam and K. Sandrasegaran, "A closed-form expression for coverage probability of random cellular network in composite rayleigh-lognormal fading channels," in Accepted for Publication at International Telecommunication Networks and Applications Conference (ITNAC), November, 2015.

[4] W. Bao and B. Liang, "Structured spectrum allocation and user association in heterogeneous cellular networks," in INFOCOM, 2014 Proceedings IEEE, April 2014, pp. 1069-1077.

[5] H.-S. Jo, Y. J. Sang, P. Xia, and J. Andrews, "Heterogeneous cellular networks with flexible cell association: A comprehensive downlink sinr analysis," Wireless Communications, IEEE Transactions on, vol. 11, no. 10, pp. 3484-3495, October 2012.

[6] S. C. Lam and K. Sandrasegaran, "Analytical coverage probability of a typical user in heterogeneous cellular networks," Journal of Networks, vol. 11, no. 2, 2016. [Online]. Available: http: //ojs.academypublisher.com/index.php/jnw/article/view/jnw11025661

[7] S. C. Lam, R. Subramanian, K. Sandrasegaran, P. Ghosal, and S. Barua, "Performance of well-known frequency reuse algorithms in lte downlink 3gpp lte systems," in Signal Processing and Communication Systems (ICSPCS), 2015 9th International Conference on, Dec 2015, pp. 1-5. 